\title{
Commentary: Fc Gamma Receptors are Expressed in the Developing Rat Brain and Activate Downstream Signaling Molecules upon Cross-Linking with Immune Complex
}

\author{
Marianna Stamou', Pamela J Lein ${ }^{2}$ \\ 'Department of Molecular Biosciences, School of Veterinary Medicine, 1089 Veterinary Medicine Drive, University of California, Davis, CA 95616 \\ ${ }^{2}$ ETH Zurich, Department of Health Sciences and Technology, Institute of Molecular Systems Biology, 8093 Zürich, Switzerland
}

\section{Article Info}

\section{Article Notes}

Received: January 24, 2019

Accepted: February 18, 2019

\section{${ }^{*}$ Correspondence:}

Dr. Pamela J. Lein, PhD, Professor of Neurotoxicology, Chair, Department of Molecular Biosciences, UC Davis School of Veterinary Medicine, Davis, CA 95616, USA; Telephone No: 530752-1970; Email: pjlein@ucdavis.edu.

(c) 2019 Lein PJ. This article is distributed under the terms of the Creative Commons Attribution 4.0 International License
During the past 20 years, a number of studies have reported IgG antibodies against viral or self-antigens in the developing human brain. Although the mechanisms by which they gain access to the developing brain are not yet clear, a subset of these antibodies has been linked to increased risk for neurodevelopmental disorders ${ }^{1-11}$. At first glance, such effects are expected to be mediated by IgG binding to its cognate Fcy receptors ( $\mathrm{Fc} \gamma \mathrm{R}$ ) on resident immune cells (such as microglia) in the brain and subsequent activation of local innate immune responses. However, considering that antibodies linked to neurodevelopmental disorders have subsequently been shown to recognize intracellular antigens expressed in neurons and astrocytes ${ }^{7}$, it is tempting to hypothesize that these autoantibodies could derail normal neurodevelopment by binding to their target antigens expressed on non-immune cells in the brain. Neuronal uptake of IgG antibodies against intracellular neuronal antigens has previously been shown and, in some cases, is thought to be mediated via clathrin-dependent endocytosis of IgG bound to Fc $\gamma$ R in these neurons ${ }^{12,13}$. In combination with recent studies showing functional expression of Fc $\gamma \mathrm{RI}$ in adult rat dorsal root ganglion neurons ${ }^{14-16}$, these findings prompted us to conduct the first comprehensive investigation of Fc $\gamma \mathrm{R}$ expression and signaling on neurons and astrocytes in the developing rat brain. In our study, we documented sex-independent in vivo expression.

In our study we documented sex-independent in vivo expression of FcgRIa, FcgRIIa, FcgRIIb, FcgRIIIa, and Fcgrt mRNA in the neonatal (postnatal days 1 and 7) rat cortex, hippocampus, and cerebellum at levels comparable to those observed in the spleen. Based on these findings, we investigated the functional expression of these Fc $\gamma \mathrm{R}$ in vitro using primary co-cultures of neurons and astrocytes from neonatal rat pups. In both hippocampal and cortical cultures, we confirmed protein expression of Fc $\gamma$ RIa, Fc $\gamma$ RIIb, and Fc $\gamma$ RIIIa but not FcRn in both cell types and showed that, similar to immune cells, expression of these receptors at the mRNA level is regulated by IFN $\gamma$. Antibody-antigen IgG immune complexes (IgG-IC) triggered increased $\left[\mathrm{Ca}^{2+}\right]_{\mathrm{i}^{\prime}}$ a small but statistically significant increase in Erk phosphorylation, and $\mathrm{Fc} \gamma \mathrm{R}$ endocytosis in these cells, consistent with canonical Fc $\gamma \mathrm{R}$ signaling in immune cells ${ }^{17-21}$. Calcium responses were concentration-dependent but showed high variability, consistent with $\left[\mathrm{Ca}^{2+}\right]_{1}$ transient variability in immune cells depending on cell type, activation state and ratio of excitatory to inhibitory Fc $\gamma \mathrm{R}$ on the cell surface ${ }^{22,23}$. The variability in $\left[\mathrm{Ca}^{2+}\right]_{\mathrm{i}}$ responses can be further explained by our findings of subsets of 
neurons and astrocytes expressing either the excitatory Fc $\gamma$ RI, or the inhibitory Fc $\gamma$ RIIb or both. Although we were not able to detect intracellular IgG in neurons or astrocytes that endocytosed Fc $\gamma \mathrm{R}$ following crosslinking with IgGIC, inhibition of Fc $\gamma \mathrm{R}$ endocytosis by pre-incubation with anti-Fc $\gamma$ RIa or anti-Fc $\gamma$ RIIb antibodies further supports that $\mathrm{Fc} \gamma \mathrm{R}$ signaling in immature neurons and astrocytes is triggered in the presence of immune complexes. Collectively, our findings of IFN $\gamma$-regulated functional $\mathrm{Fc} \gamma \mathrm{R}$ expression by developing neurons and astrocytes underscore the need for further investigation into the role of $\mathrm{Fc} \gamma$ receptors during normal and aberrant neurodevelopment.

The strength of our experimental approach is the functional characterization of neuronal/astrocytic Fc $\gamma \mathrm{R}$ using IgG-IC to crosslink Fc $\gamma$ R on the cell surface and activate downstream signaling. Although IgG glycosylation is an important regulator of IgG-mediated immune signaling, monosaccharide IgG antibodies can also mediate effector functions in immune cells via $\mathrm{Fc} \gamma \mathrm{R}$, but in vitro these functions can be predicted only when immune complexes are used ${ }^{24}$. In addition, with the exception of Fc $\gamma$ RI, which can be activated by monomeric IgG, all other IgG subclasses are activated in the presence of immune complexes but not by monomeric IgG (with the exception of FcRn, which functions to protect monomeric IgG from degradation) ${ }^{18,}$ 25 . To promote activation of all Fc $\gamma \mathrm{R}$ isoforms in our study, and considering that we had no information regarding the glycosylation state of the IgG used to generate the immune complexes, we pre-incubated mouse IgG with rat anti-mouse IgG to generate IgG-IC across all our functional studies.

Despite this strength, there are potential gaps in our findings. Although the downstream effects we observed in our study (increased $\left[\mathrm{Ca}^{2+}\right]_{i}$, Erk phosphorylation and $\mathrm{Fc} \gamma \mathrm{R}$ endocytosis) were triggered in the presence of IgGIC, the IgG-IC concentration used to observe endocytosis was above physiologically relevant levels documented in rat cerebrospinal fluid $(100 \mu \mathrm{g} / \mathrm{ml}$; although in humans such levels would be considered low $)^{26-28}$, while similarly high concentrations of monomeric IgG also triggered $\left[\mathrm{Ca}^{2+}\right]_{\mathrm{i}}$ and Erk phosphorylation but not Fc $\gamma \mathrm{R}$ endocytosis. At the same time, the exact composition of the IgG-IC (ratio between rat IgG subclasses, IgG glycosylation state, and size of the immune complex) was not characterized and could have varied between individual preparations, thus affecting Fc $\gamma \mathrm{R}$ crosslinking, activation and downstream signaling ${ }^{24,}$ 26,29 . Another consideration is that the Fc portion of IgG can also be recognized by non-classical Fc-binding receptors on the cell surface. Such receptors have been described in immune cells (FcR-like 5 (FcRL5), Dectin-2, DC-Sign, MMR and MBL2) ${ }^{30}$, but the expression of such molecules in neurons/astrocytes in the developing brain has not yet been investigated. In summary, the specificity of $F c \gamma R$ involvement in the IgG-IC-induced cellular signaling should be confirmed by knocking down FcyR expression in these cells; this would also be useful for further assessing the specificity of the commercially available antibodies used to probe Fc $\gamma$ R protein expression in these cells. Studies of IgGIC-mediated activation of additional signaling molecules downstream of Fc $\gamma \mathrm{R}$ should also be conducted to identify alignment with or potential deviations from canonical immune cell Fc $\gamma$ R signaling, which would further confirm a functional role of Fc $\gamma \mathrm{R}$ in developing neurons/astrocytes. As an example, activation of neuronal Fc $\gamma \mathrm{RI}$ in rat DRG neurons (which express Fc $\gamma$ RIa but not Fc $\gamma$ RIIb) results in increased $\left[\mathrm{Ca}^{2+}\right]_{\mathrm{i}}$ by triggering the non-selective cation channel TRPC3 through the Syk-PLC-IP ${ }_{3}$ pathway ${ }^{15,16}$. Although we probed for Syk phosphorylation in rat hippocampal and cortical neurons, we were not able to demonstrate Syk expression in these cells (unpublished data). The possibility that manual application of compounds to cultured neurons may trigger mechanosensitive receptor-mediated $\left[\mathrm{Ca}^{2+}\right]_{\mathrm{i}}$ increase ${ }^{31}$, thus partially contributing to the observed effects, cannot be excluded. In addition, considering that hippocampal and cortical neurons express activating and inhibitory Fc $\gamma \mathrm{R}$ on their surface, isoform-specific Fc $\gamma \mathrm{R}$ knockdown and careful probing of downstream effectors would be useful in dissecting the relative contribution of each isoform on the downstream pathways activated ${ }^{32-34}$.

But what if the true role of neuronal/astrocytic Fc $\gamma \mathrm{R}$ lies in bidirectional signaling with resident microglia and other immune cells in the brain? Expression of classical immune molecules such as MHC Class I proteins and complement receptors has been demonstrated to regulate microgliamediated synaptic pruning during neurodevelopment ${ }^{35-37}$. Generating conditional neuronal $\mathrm{F} c \gamma \mathrm{R}$ knockout models would be instrumental in assessing the existence of analogous interactions between neuronal $\mathrm{Fc} \gamma \mathrm{R}$ and microglia, and in understanding the role of neuronal Fc $\gamma \mathrm{R}$ in vivo.

Going back to the original question of whether autoantibodies against intracellular brain antigens could derail normal neurodevelopment by engaging their intracellular targets via Fc $\gamma$ R-mediated uptake, there are additional questions that need to be addressed. Are these autoantibodies taken up by developing neurons in a non-stochastic manner? Are these autoantibodies already complexed with their target antigens when internalized? If not, is there another mechanism for their uptake into neurons which does not involve Fc $\gamma \mathrm{R}$ engagement? Are these autoantibodies function-blocking? Alternatively, considering that non-specific monomeric IgG may be neuroprotective ${ }^{26}$, could this be the case also for maternal autoantibodies with specific brain antigen targets? Or are these autoantibodies simply diagnostic? Would neuronal subtypes with prevalent Fc $\gamma$ RI expression be more susceptible to uptake and effects of these 
antibodies? How would endocytosed IgG escape lysosomal degradation in neurons? Further studies addressing these questions are needed in order to assess a potential role for $\mathrm{Fc} \gamma \mathrm{R}$ in developing neurons and astrocytes in the reported association between maternal antibodies targeting developing brain antigens and increased risk for neurodevelopmental disorders.

In summary, hippocampal and cortical neurons express $\mathrm{Fc} \gamma$ receptors and IgG immune complexes seem to crosslink these receptors, resulting in activation of downstream signaling. Although the functional readouts of IgG-IC priming of these cells are part of the immune cell signaling cascade normally activated by $\mathrm{Fc} \gamma \mathrm{R}$ crosslinking, further studies are needed to further elucidate the neurodevelopmental consequences of $\mathrm{Fc} \gamma \mathrm{R}$ expression in developing neurons and astrocytes and probe their potential activation by maternal autoantibodies against brain antigens.

\section{References}

1. Strous RD, Shoenfeld Y. Schizophrenia, autoimmunity and immune system dysregulation: a comprehensive model updated and revisited. Journal of autoimmunity. 2006; 27: 71-80.

2. Brown AS. The environment and susceptibility to schizophrenia. Progress in neurobiology. 2011; 93: 23-58.

3. Mortensen PB, Pedersen CB, Hougaard DM, et al. A Danish National Birth Cohort study of maternal HSV-2 antibodies as a risk factor for schizophrenia in their offspring. Schizophrenia Research. 2010; 122: 257-263.

4. Fox-Edmiston E, Van de Water J. Maternal Anti-Fetal Brain IgG Autoantibodies and Autism Spectrum Disorder: Current Knowledge and its Implications for Potential Therapeutics. CNS drugs. 2015; 29 715-724.

5. Dalton P, Deacon R Fau - Blamire A, Blamire A Fau - Pike M, et al. Maternal neuronal antibodies associated with autism and a language disorder.

6. Zimmerman AW, Connors Sl Fau - Matteson KJ, Matteson Kj Fau - Lee LC, et al. Maternal antibrain antibodies in autism.

7. Braunschweig D, Krakowiak P, Duncanson P, et al. Autism-specific maternal autoantibodies recognize critical proteins in developing brain. Translational psychiatry. 2013; 3: e277.

8. Martinez-Cerdeno V, Camacho J, Fox E, et al. Prenatal Exposure to Autism-Specific Maternal Autoantibodies Alters Proliferation of Cortical Neural Precursor Cells, Enlarges Brain, and Increases Neuronal Size in Adult Animals. Cerebral cortex. 2016; 26: 374-383.

9. Bauman MD, Iosif Am Fau - Ashwood P, Ashwood P Fau - Braunschweig D, et al. Maternal antibodies from mothers of children with autism alter brain growth and social behavior development in the rhesus monkey.

10. Braunschweig D, Ashwood P Fau - Krakowiak P, Krakowiak P Fau Hertz-Picciotto I, et al. Autism: maternally derived antibodies specific for fetal brain proteins.

11. Nordahl CW, Braunschweig D Fau - Iosif A-M, Iosif Am Fau - Lee A et al. Maternal autoantibodies are associated with abnormal brain enlargement in a subgroup of children with autism spectrum disorder.

12. Greenlee JE, Clawson SA, Hill KE, et al. Neuronal uptake of anti-Hu antibody, but not anti-Ri antibody, leads to cell death in brain slice cultures. Journal of neuroinflammation. 2014; 11: 160.
13. Congdon EE, Gu J, Sait HBR, e al. Antibody Uptake into Neurons Occurs Primarily via Clathrin Dependent Fc $\gamma$ Receptor Endocytosis, and is a Prerequisite for Acute Tau Clearance. Journal of Biological Chemistry. 2013.

14. Qu L, Zhang $\mathrm{P}$, LaMotte $\mathrm{RH}$, et al. Neuronal Fc-gamma receptor I mediated excitatory effects of IgG immune complex on rat dorsal root ganglion neurons. Brain behavior and immunity. 2011; 25: 1399 1407.

15. Qu L. Neuronal Fc gamma receptor I as a novel mediator for IgG immune complex-induced peripheral sensitization. Neural regeneration research. 2012; 7: 2075-2079.

16. $\mathrm{Qu} \mathrm{L}$, Li Y, Pan $\mathrm{X}$, et al. Transient receptor potential canonical 3 (TRPC3) is required for IgG immune complex-induced excitation of the rat dorsal root ganglion neurons. The Journal of neuroscience : the official journal of the Society for Neuroscience. 2012; 32: 9554-9562.

17. Stamou M, Grodzki AC, van Oostrum M, et al. Fc gamma receptors are expressed in the developing rat brain and activate downstream signaling molecules upon cross-linking with immune complex. Journal of neuroinflammation. 2018; 15: 7-7.

18. Nimmerjahn F, Ravetch JV. Fcgamma receptors as regulators of immune responses. Nature reviews Immunology. 2008; 8: 34-47.

19. Young JD, Ko SS, Cohn ZA. The increase in intracellular free calcium associated with IgG gamma $2 \mathrm{~b} /$ gamma 1 Fc receptor-ligand interactions: role in phagocytosis. Proceedings of the National Academy of Sciences of the United States of America. 1984; 81: 54305434 .

20. Myers JT, Swanson JA. Calcium spikes in activated macrophages during Fcgamma receptor-mediated phagocytosis. Journal of leukocyte biology. 2002; 72: 677-684.

21. Lucas $M$, Zhang $X$, Prasanna V, et al. ERK activation following macrophage FcgammaR ligation leads to chromatin modifications at the IL-10 locus. Journal of immunology. 2005; 175: 469-477.

22. Vig M, Kinet JP. Calcium signaling in immune cells. Nature immunology. 2009: 10: 21-27.

23. Oh-hora M. Calcium signaling in the development and function of T-lineage cells. Immunological reviews. 2009; 231: 210-224.

24. Kao D, Danzer H, Collin M, et al. A Monosaccharide Residue Is Sufficient to Maintain Mouse and Human IgG Subclass Activity and Directs IgG Effector Functions to Cellular Fc Receptors. Cell Reports. 2015; 13: 2376-2385.

25. Simister NE, Mostov KE. An Fc receptor structurally related to MHC class I antigens. Nature. 1989; 337: 184-187

26. Hulse RE, Swenson WG, Kunkler PE, et al. Monomeric IgG is neuroprotective via enhancing microglial recycling endocytosis and TNF-alpha. The Journal of neuroscience : the official journal of the Society for Neuroscience. 2008; 28: 12199-12211.

27. Furst DE. Serum Immunoglobulins and Risk of Infection: How Low Can You Go? Seminars in Arthritis and Rheumatism. 2009; 39: 18-29.

28. Dati F, Schumann G Fau - Thomas L, Thomas L Fau - Aguzzi F, et al. Consensus of a group of professional societies and diagnostic companies on guidelines for interim reference ranges for 14 proteins in serum based on the standardization against the IFCC/BCR/CAP Reference Material (CRM 470). International Federation of Clinical Chemistry. Community Bureau of Reference of the Commission of the European Communities. College of American Pathologists.

29. Lux A, Yu X, Scanlan CN, et al. Impact of Immune Complex Size and Glycosylation on IgG Binding to Human Fc $\gamma$ Rs. The Journal of Immunology. 2013; 1200501.

30. Boesch AW, Brown EP, Cheng HD, et al. Highly parallel characterization of IgG Fc binding interactions. mAbs. 2014; 6: 915-927. 
31. Gu Y, Gu C. Physiological and pathological functions of mechanosensitive ion channels. Molecular neurobiology. 2014; 50: 339-347.

32. Guilliams M, Bruhns P, Saeys Y, et al. The function of Fcgamma receptors in dendritic cells and macrophages. Nature reviews Immunology. 2014; 14: 94-108.

33. Rosales C, Uribe-Querol E. Fc receptors: Cell activators of antibody functions. Advances in Bioscience and Biotechnology. 2013; 04: 21 33.

34. Hogarth PM, Pietersz GA. Fc receptor-targeted therapies for the treatment of inflammation, cancer and beyond. Nature reviews Drug discovery. 2012; 11: 311-331.

35. Brown GC, Neher JJ. Microglial phagocytosis of live neurons. Nature reviews Neuroscience. 2014; 15: 209-216

36. Bialas AR, Stevens B. TGF- $\beta$ signaling regulates neuronal C1q expression and developmental synaptic refinement. Nature neuroscience. 2013; 16: 1773.

37. Lee H, Brott BK, Kirkby LA, et al. Synapse elimination and learning rules co-regulated by MHC class I H2-Db. Nature. 2014; 509: 195. 